\title{
Tendencias y efectos socioambientales del desarrollo inmobiliario turístico en zonas costeras de Sudamérica. El caso de Región Este, Uruguay
}

Isabel Gadino. Universidad de la República, Maldonado, Uruguay. José Sciandro. Universidad de la República, Maldonado, Uruguay. Germán Taveira. Universidad de la República, Maldonado, Uruguay. Nathalie Goldberg. Universidad de la República, Maldonado, Uruguay

RESUMEN | La costa de Región Este, Uruguay, tiene un desarrollo histórico vinculado al turismo residencial. En las últimas décadas aprobó nuevos instrumentos de ordenamiento territorial. Simultáneamente, el avance inmobiliario, en particular el destinado al turismo neoexclusivo (TNE), se extiende y acapara espacios naturales. Este trabajo analiza resultados locales del TNE, comparando la apropiación de servicios ecosistémicos y las dinámicas socioterritoriales, con el modelo de costa promovido desde el ordenamiento territorial y la legislación vinculada. Se contrasta información georreferenciada con análisis cuantitativos y cualitativos de normativas, solicitudes de permisos ambientales, solicitudes de excepciones al ordenamiento territorial e información empleada para la oferta de los emprendimientos en el mercado. Se observa una clara relación entre sitios ecosistémicos clave y los nuevos emprendimientos, grandes discordancias en las informaciones emitidas por los promotores, así como un claro sesgo de la normativa tendiente al uso exclusivo del espacio en aras de su conservación.

PALABRAS CLAVE | ordenamiento territorial, desarrollo sustentable, barrios cerrados.

ABSTRACT | The coast of East Region, Uruguay, has a historical development linked to residential tourism. In recent decades, new territorial planning instruments have been approved, while real estate development, particularly that destined to neo-exclusive tourism (NET), is expanding and taking over natural areas. This paper studies local results of NET, comparing the appropriation of ecosystem services and the socio-territorial dynamics, with the coastal model promoted from the territorial ordering and its related legislation. Georeferenced information is contrasted with quantitative and qualitative analyses of regulations, applications for environmental permits, requests for exceptions to territorial planning, and information used by real estate sale sites. There is a clear relationship between key ecosystems and the new developments, great discrepancies in the information issued by the promoters, as well as a clear bias of the regulations tending to the exclusive use of the space for the sake of its conservation.

KEYWORDs | spatial planning, sustainable development, gated communities. 


\section{Introducción}

Durante las primeras décadas del siglo xx, gran parte de la costa de Región Este (Figura 1) fue fraccionada en pequeños lotes y transformada de rural a urbana, como estrategia para aunar la producción especulativa de suelo (Harvey, 2014) con el turismo de sol y playa. Desde entonces, la zona ha estado asociada al turismo residencial (TR), ese conjunto de prácticas sociales que giran en torno a la producción de segundas residencias, infraestructuras, servicios y espacios vinculados, y cuyo uso está mayoritariamente ligado a la esfera del ocio y no a las de producción y reproducción (Aledo, 2016). A lo largo de este territorio -con valores más altos en el departamento de Maldonado que en Rocha-, la cantidad de viviendas supera ampliamente el número de pobladores permanentes (Robayna, 2009). Sin embargo, la consolidación histórica de cada balneario fraccionado ha sido despareja, dependiendo principalmente de la cercanía y accesibilidad desde ciudades emisoras de los potenciales propietarios y de la aptitud para baño de sus playas. La situación económica del país y de Argentina -cuyas clases medias altas tienen un rol fundamental en este desarrollo (Díaz Pellicer, 2012; Trochon, 2017)- fue determinante para el éxito o fracaso de la venta de los loteos en Uruguay. A principios de los años 2000, buena parte de los fraccionamientos rochenses y algunos sectores en Maldonado se mantenían agrestes o con escaso desarrollo urbano (Roche \& Gadino, 2018).

\section{FIGURA I | Ubicación del área de estudio y áreas de turismo neoexclusivo (TNE)}

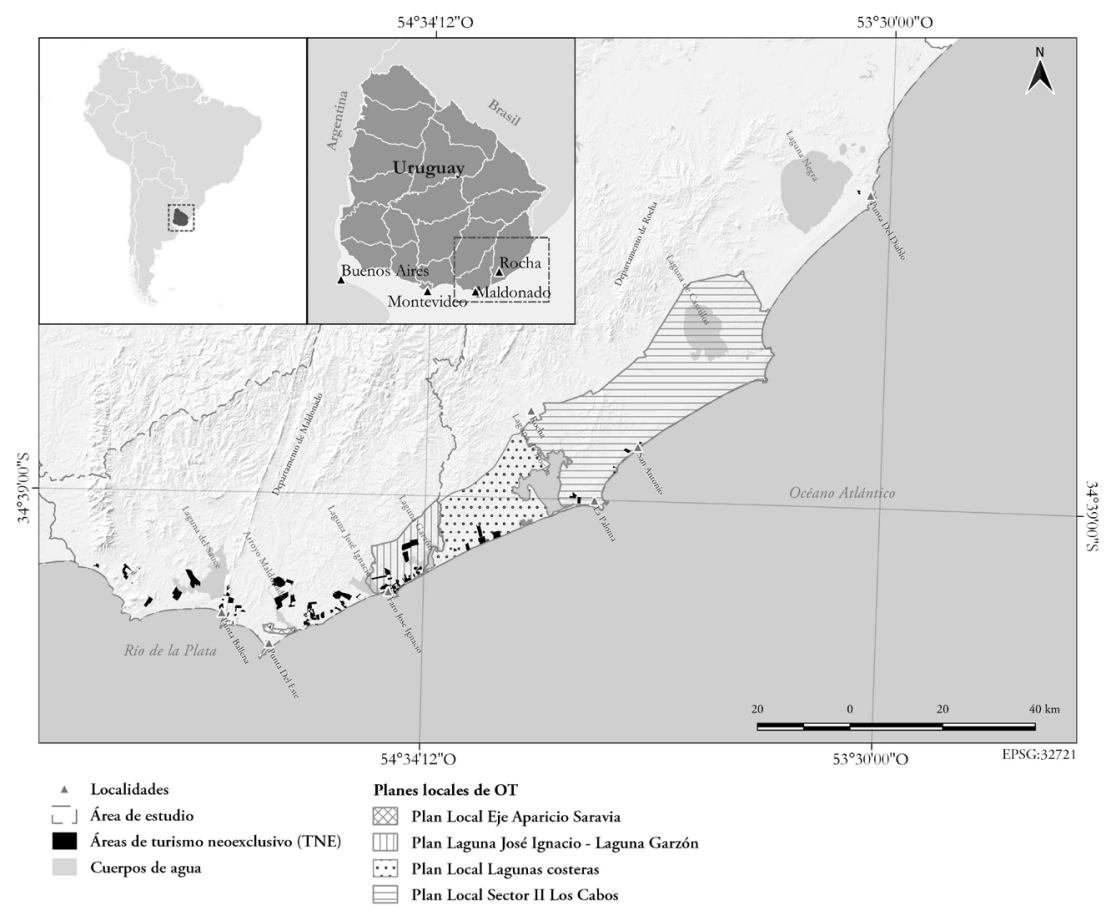

FUENTE: ELABORACIÓN PROPIA 
En las últimas décadas, de igual forma que en remanentes rurales costeros, se observa la expansión de barrios cerrados, fenómeno cuestionado desde diversos ámbitos de análisis urbano por generar graves consecuencias sobre el tejido social, relacionadas a la fragmentación y segregación socioespacial, polarización, disuasión de interacciones entre grupos sociales diferentes y aumento de las desigualdades (Lang \& Danielsen, 1997; Mendonça Ferreira \& Villas Boas, 2020; Roitman, 2013; Svampa, 2005). En paralelo aparecen condominios horizontales y torres "all inclusive", que sustituyen antiguas edificaciones en primera línea de destinos turísticos maduros, incorporando procesos de guetización en la ciudad abierta.

La consolidación de estas nuevas tipologías cerradas asociadas al turismo impacta fuertemente en España (Aledo, 2008; Domínguez \& Aledo, 2005), América Central y Sudamérica (Baptista et al., 2018; Camacho Lomelí, 2015; Clement \& Grant, 2012). Demajorovic et al. (2011) observan que, en el caso de Sudamérica, este modelo de desarrollo en constante consumo de recursos naturales es excluyente, dualista y privatizador; ha generado valoración del precio de la tierra, aumento de procesos especulativos, afectación a la economía y cultura locales, y entiende los procesos de planificación como obstáculo al crecimiento. Por su lado, Hernández (2010) identifica un nuevo modelo urbano turístico de carácter exclusivo y selectivo, que se diferencia de las tipologías empleadas hasta principios del siglo xx por poseer nuevas formas culturales de sociabilización (p.e., deportes náuticos, uso de vehículos todoterreno), autosegregación espacial, interacción y transformación del medio natural, y por el uso de una estrategia de venta muy ligada a la apropiación de la naturaleza como valor de mercado, al prestigio y la exclusividad. El autor denomina a este fenómeno como "turismo neoexclusivo" (TNE).

Trabajos recientes en Europa, Centro y Sudamérica, Asia y África, mencionan que la participación de grandes capitales globales en esta nueva modalidad de producción inmobiliaria (sensu Harvey, 2014), sumada a las facilidades otorgadas por gobiernos nacionales y locales para posibilitar estas inversiones, producen ganancias en general asociadas con la capacidad especulativa internacional más que con un desarrollo sustentable local (Aizcorbe et al., 2014; Babinger, 2012; García de Fuentes et al., 2019; Hidalgo et al., 2014; Kranjčević \& Hajdinjak, 2019; Morell \& Membrado-Tena, 2019; Pontes et al., 2020; Sousa et al., 2016; Van Noorloos, 2015). Mientras tanto, se evidencia el distanciamiento entre los desarrollos reales y las representaciones y escenificaciones oficiales, empleadas para posicionar estos territorios en el mercado turístico global como producto natural (Barboza Núñez, 2019). Sumado a lo anterior, la reciente crisis generada por la pandemia de Covid-19 deja manifiesta la gran vulnerabilidad social y económica que, frente a situaciones de este tipo, a nivel mundial presentan los sitios con fuerte dependencia del turismo (Félix \& García, 2020; Pitarch-Garrido, 2020; Zielinski \& Botero, 2020). ${ }^{1}$

Las relaciones entre sociedad y naturaleza, en particular las vinculadas a la sobreexplotación de bienes y de servicios ecosistémicos (sSEE) (Millennium Ecosystem Assessment [MEA], 2005), incorporación de nuevos recursos en el mercado o

1 Según cifras oficiales de Uruguay (Ministerio de Turismo [Mintur], 2021), en enero 2021 las llegadas internacionales cayeron $97 \%$, mientras el turismo interno creció del $30 \%$ al $50 \%$. 
distribución de beneficios, son temas de especial interés para la Geografía contemporánea o la Ecología Política. Esta última busca comprender las formas de pensar la naturaleza, entendiendo que los discursos son capaces de producir y reproducir relaciones de poder desequilibradas (Leff, 2015), y que las luchas en torno al uso de los recursos no son solo materiales, produciéndose también en relación con los significados (De Matheus e Silva et al., 2018; Escobar, 1995, 2000, citados en Durand Smith et al., 2011).

Las políticas neoliberales han forjado un modelo de regulación de la naturaleza y conservación de la biodiversidad (Igoe \& Brockington, 2007), que tiene un componente pragmático con origen en la crítica a la gestión burocrática e ineficaz del Estado en la década de 1970 (Beck, 2013), y resulta fuente de inspiración del informe Brundtland (1987) y del concepto de desarrollo sustentable. Este enfoque sostiene que no tiene por qué haber incompatibilidad en el binomio ambientedesarrollo económico, sino que existen soluciones capaces de favorecer a ambos (March, 2013). Los antecedentes de este vínculo se pueden sintetizar en el discurso sobre la modernización ecológica, el presunto fracaso de la gobernanza ambiental y los costes de regulación (McCarthy \& Prudham, 2004); así, el mercado se ha abierto camino en la gestión ambiental (Jordan et al., 2003). De manera análoga, la neoliberación de la naturaleza pretende la unión entre protección ambiental, crecimiento económico y eficiencia (Brockington \& Igoe, 2006). El establecimiento de derechos de propiedad, la utilización de mercados como mecanismos de asignación, la colaboración público-privada y la internalización de las externalidades ambientales a través del precio, son los principales dispositivos descritos (March, 2013).

Castree (2006) describe el proceso de privatización como la asignación de derechos de propiedad sobre fenómenos ambientales o sociales antes controlados por el Estado o la comunidad, o no controlados en lo absoluto. De acuerdo con el MEA (2005), "los beneficios no comercializados a menudo se pierden o se degradan. Estos beneficios no comercializados son a menudo altos y a veces más valiosos que los comercializados" (p. 6). Los defensores de esta tesis sostienen que fijar el precio de los procesos naturales ayudará a sostenerlos, y que estos sistemas serían explotados de peores maneras sin esta internalización. Para Gómez-Baggethun et al. (2010) estas visiones proponen la incorporación a la economía formal de todos los ecosistemas y procesos biofísicos, los que se redefinen en términos de valor de uso social (SSEE); para dichos autores, ello facilita y orienta la toma de decisiones con una visión que ayuda a la conservación del ambiente. Scott (1998), por su parte, sostiene que los nuevos mercados se abstraen de las complejidades inherentes a los sistemas naturales que intentan regular y comercializar, puesto que la naturaleza no admite esta simplificación. Argumenta que las clasificaciones fallidas que se utilizan para comercializar la naturaleza en un marco jurídico pueden generar fallas que, en definitiva, degradan los ecosistemas involucrados.

En las últimas tres décadas se formularon críticas al descubrimiento y la extracción de recursos, basadas en el concepto de economía verde (Peet \& Watts, 2002; Peluso \& Lund, 2011; Robbins, 2012) y se analizan las consecuencias de la aplicación de las ideas ambientales neoliberales en diversos lugares (Harvey, 1989; McCarthy \& Prudham, 2004; Peck \& Tickell, 2002). Las opiniones contra esta ampliación 
de fronteras neoliberal refieren a que la riqueza asociada a estas modalidades no proviene del crecimiento económico real, la dinámica del valor del trabajo o las rentas tecnológicas asociadas a la producción industrial, sino de la apropiación de la riqueza socionatural de "fuera" del sistema capitalista industrial existente (Fairhead et al., 2012; McAfee, 1999). Para Knuth (2016), el ambientalismo de mercado está siendo cada vez más utilizado en el ordenamiento territorial.

\section{Antecedentes locales}

Previamente a su loteo masivo, la costa de Región Este formaba parte de grandes estancias dedicadas a la ganadería extensiva. En este contexto, playa, arenales y lagunas costeras eran considerados inservibles, por lo que la iniciativa de transformar la zona litoral a urbana fue tomada como positiva por propietarios, autoridades y técnicos relacionados principalmente con el sector inmobiliario. De acuerdo con la legislación que amparó esta transformación (Ley de Centros Poblados, 1946), solamente los primeros 150 metros a partir de la línea de ribera se destinaron a espacio público, mientras el resto del área, incluyendo extensos campos de dunas móviles de hasta 40 metros de altura (FREPLATA, 2005), quedó loteada y pasó a pertenecer a múltiples propietarios.

Casi un siglo de desarrollo urbano turístico ha generado en Región Este impactos que se observan como positivos. Si bien las diferencias demográficas son muy amplias, ${ }^{2}$ para el área se cuentan: i) $20 \%$ del total de puestos de trabajo vinculados al sector turístico en todo el país, el cual aporta un 8,6\% al PBI nacional (Ministerio de Turismo [MINTuR], 2018); ii) inversiones y trabajos en la industria de la construcción: en 2012 se contabilizaron 12.000 puestos de trabajo y 615.000 metros cuadrados aprobados en Maldonado (El Observador, 2018); y iii) el disfrute del sitio por parte de la clase media y alta.

Como negativos, se marca la elevada estacionalidad del tipo de actividad hegemónica (Robayna, 2009), además de numerosos daños en sseE, como afectación y pérdida de biodiversidad (Soutullo et al., 2013) y fragmentación de hábitats (Gadino et al., 2012). Alonso y Bassagoda (2002) advierten sobre el riesgo de desaparición completa de parte de la flora costera del Uruguay, señalando, por ejemplo, que sobre una franja litoral de un kilómetro de ancho se encuentra un tercio del total de especies de árboles y arbustos del país. Numerosos estudios demuestran el deterioro de la capacidad disipadora de playas con incremento de erosión y retroceso de línea de costa (Boretto et al., 2018; de Álava, 2008; Oficina de Planeamiento y Presupuesto [opp], Organización de los Estados Americanos [oEA] \& Banco Interamericano de Desarrollo [BID], 1992; Panario \& Gutiérrez, 2005), así como pérdida de sitios arqueológicos costeros (Brum Bulanti et al., 2020). A esto debe anexarse las previsiones de mayor vulnerabilidad litoral frente a futuros escenarios de subida del nivel medio del mar (Nagy et al., 2016). En materia paisajística, la Dirección

2 Según el Instituto Nacional de Estadística y Censo (2011), Maldonado tiene una población de 164.000 habitantes con una de las tasas de crecimiento poblacional más alta del país, mientras que Rocha tiene 68.000 habitantes y su población decrece. 
Nacional de Ordenamiento Territorial (DINOT, 2011) señala la caricaturización que sufren los paisajes de la zona.

Para Conde (2014), lo anterior evidencia el acumulado de excesiva planificación sectorial relacionada con el turismo, la urbanización, la actividad portuaria, la infraestructura industrial y la conservación (áreas protegidas), que crea competencias inviables por los recursos naturales. Sobre el tema, Castro Casas (2018) observa que, en los últimos treinta años, el orden jurídico uruguayo ha generado un proceso de descubrimiento del ordenamiento territorial, dejando atrás una situación con múltiples normas dispersas. El autor señala, como hito en este proceso, la aprobación de la Ley de Ordenamiento Territorial y Desarrollo Sostenible (LOTds, 2008), que proporciona normas de fondo sobre la materia y el derecho de propiedad sobre el suelo, a la vez que abre la posibilidad a diversos instrumentos de ordenación. Durante su proceso de aprobación y a partir de ella, relacionado con el espacio de estudio se generaron las Estrategias Regionales de Ordenamiento Territorial y varios instrumentos a cargo de los gobiernos departamentales; entre ellos, las directrices propias de cada departamento, la nueva categorización de suelos y varios planes locales. Este nuevo conjunto de herramientas se suma a otras de orden nacional, como la Evaluación de Impacto Ambiental, la que en ámbitos costeros ha dado resultados cuestionables (Gadino \& Taveira, 2020).

En paralelo, se visualiza el crecimiento explosivo de urbanizaciones cerradas dispersas, aunque mayoritariamente sin ocupación real. En este caso, las tipologías principales son: barrio privado, club de campo, chacras marítimas y complejo más casas (García, 2019; Varela, 2017). Simultáneamente se constata la aparición de complejos edilicios exclusivos en los centros urbanos consolidados, observándose el desarrollo del TNE en todo el litoral regional. Se agrega a esto la apertura de una política de exoneración tributaria y flexibilización urbana que aplica la Intendencia Departamental de Maldonado desde fines de 2015, cuyo objetivo principal es fomentar la industria de la construcción. Varios de los casos aprobados en este régimen de excepciones son emprendimientos destinados al turismo de altos ingresos.

Junto con lo anterior, buena parte de la costa no demarcada y/o consolidada no solo contribuye a la conservación de la zona litoral activa, sino que se usa y percibe por la población como porciones del espacio público de la playa, hecho que genera múltiples conflictos cuando se anuncian obras para su transformación por parte de privados.

En este contexto nos preguntamos: ¿qué variables locales presenta la planificación para el desarrollo sustentable de ambientes costeros?, ¿qué servicios ecosistémicos quedan involucrados y cómo, con los usos promovidos?, ¿están siendo empleados los instrumentos legales en todo su potencial? Este trabajo contrasta el modelo teórico de ordenamiento territorial nacional y local de Región Este, Uruguay, con casos de TNE construidos. Se busca exponer contradicciones, fallas y coincidencias del sistema, que favorezcan un análisis crítico y mejoras enfocadas hacia una gobernanza socioambiental. 


\section{Estrategia metodológica}

A partir del marco teórico de la Ecología Política, se estudian las diferentes partes del proceso de desarrollo del TNE en la Región Este, desde la formalización jurídica de la planificación territorial hasta su concreción material. En este contexto se desarrolla una cadena de explicación (Robbins, 2012) que permite identificar los diferentes actores y sus discursos en torno a un problema socioterritorial, y las dinámicas económicas y políticas que tienen repercusiones en la degradación local de los recursos. Se emplean métodos deductivos y cualitativos para el estudio de documentos: planes, directrices, solicitudes de aprobación de proyectos, solicitudes de excepciones y páginas de venta. Los resultados en el territorio se analizan a través de sistemas de información geográfica y verificaciones en campo, en particular superficies ocupadas y ubicación. Finalmente se reflexiona sobre los espacios normativoculturales que sustentan el TNE local.

El área de estudio es una faja paralela a la costa de Maldonado y Rocha, de aproximadamente 10 kilómetros de ancho. Esta delimitación está relacionada con el espacio comprendido por los planes locales analizados y la ubicación de los emprendimientos que se consideran representativos del TNE. Existe normativa nacional común para ambos departamentos, y además cada uno tiene autonomía legislativa para ordenar su territorio. Como se ha señalado tanto en textos académicos como técnicos -incluyendo los propios instrumentos analizados-, sumados a los SSEE culturales relacionados al turismo, en el área se mantienen espacios proveedores de otros servicios clave para la sustentabilidad en la Región Este. Entre ellos se cuentan: el abastecimiento de agua potable del departamento de Maldonado (Laguna del Sauce y Laguna Blanca); el aprovisionamiento de alimento a población local y turismo (suelos productivos y cuerpos de agua en general); la regulación de inundaciones, subida del nivel medio del mar y erosión (humedales y sistema dunar); y el soporte para hábitats de especies en muchos casos endémicas (humedales, puntas rocosas, sierras, lagunas costeras, cordones dunares).

Para esta área:

Se revisa la normativa de ordenamiento y gestión, aprobada a partir de la nueva concepción del ordenamiento territorial (От):

a. Maldonado_ Directriz Departamental, Plan Local de от para la protección y desarrollo sostenible del área entre las lagunas de José Ignacio y Garzón desde la Ruta 9 al océano Atlántico (Entre Lagunas), Plan Local de от Eje Av. Aparicio Saravia de Maldonado - Punta del Este.

b. Rocha_ Directriz Departamental, Plan de от у Desarrollo Sustentable de la Costa Atlántica del Departamento de Rocha (Ordenanza Costera [oc]), Plan Parcial de OT Lagunas Costeras, Plan Local de от Los Cabos.

Usando la Teoría Fundamentada (Corbin \& Strauss, 1990), se detectan los principales códigos que emergen de estos documentos y permiten encontrar los supuestos fundantes. Se procesa con el programa Atlas Ti v8 para analizar jerarquía y relaciones entre códigos mediante el mapeo de redes semánticas, la exploración de las 
coocurrencias entre estos y, finalmente, las palabras o conceptos que se refuerzan por repetición asociados a las mismas categorías.

Se analiza información espacial (imágenes satelitales e información georreferenciada):

a. Se relevan los emprendimientos pertenecientes al TNE asociados a la costa de Región Este, determinados con base en: i) datos públicos del instrumento Programa de Actuación Integrada (PAI), empleado para transformar suelo rural a urbano o suburbano con destino turístico, y de los instrumentos Viabilidades de Localización (VAL) e Informes Ambientales Resumen (IAR), usados para comunicar cómo se desarrollará el fraccionamiento una vez transformado el suelo; ii) información de solicitudes de excepciones al от aprobadas por la Junta Departamental de Maldonado (JDM) entre 2015 y 2019; y iii) cruzamiento y/o verificación a partir de imagen satelital, prensa y/o sitio. Para cada caso se analiza ubicación, área, extensión, fecha de aprobación y planificación territorial vinculada. Finalmente, cada emprendimiento se clasifica, de acuerdo con su despliegue en el territorio, por tipologías: barrio, condominio o edificio.

b. Se analiza la relación de la capa de TNE con una capa de áreas de interés ambiental (AIA). Esta se basa en la capa de usos de suelo relevados en 2015 (Álvarez et al., 2015), a la que se agregan zonas de provisión de SSEE relativos al TNE. Estos son los categorizados como servicios culturales, que involucran la recreación, el turismo y valoración estética del paisaje, entre otros (Daniel et al., 2012). La composición final de esta capa es la sumatoria de usos correspondientes a aguas naturales, área natural inundable, áreas desnudas (arena de playa), monte nativo y palmares.

c. Se analiza la relación AIA - TNE, a través de un análisis de distancia ráster en software QGIS 3.10 desde las AIA. Se intersecta el ráster de distancias con las áreas de TNE y se calculan estadísticos descriptivos a partir del promedio de distancias a cada punto de la superficie de las áreas de TNE. Se calculan áreas de superficie y distancias de borde solapadas entre ambas capas.

d. Se comparan áreas de padrones urbanos ubicados dentro del área de estudio con la superficie total de TNE relevado.

Se analizan las solicitudes de aprobación oficial de emprendimientos, y se comparan con datos de sus sitios de promoción, enfocando en la venta de SSEE:

Se analiza el contenido de los PaI, las val y los IAR disponibles. En paralelo, se analiza la información de sitios de promoción y venta de los emprendimientos. Se rastrean textos e imágenes relacionados con la condición de emprendimiento cerrado, los SSEE ofrecidos y el vínculo entre estos y las nociones de prestigio y exclusividad.

Se analiza la politica de excepciones en el departamento de Maldonado:

Se estudian los expedientes de la Junta Departamental de Maldonado (JDM) donde se aprueban las solicitudes de excepciones (denominados administrativamente "consultas de viabilidad"). Se revisan cuántas de estas aprobaciones refieren al TNE, a qué tipología y en qué consisten los permisos otorgados. Se 
comparan datos con análisis de porcentaje de excepcionalidad realizado por la Dirección Nacional de Ordenamiento Territorial (DINOT, 2018).

\section{Resultados}

\section{El marco de la normativa territorial}

La planificación territorial desarrollada para el sector costero mantiene los objetivos de la legislación de mayor rango, nacional y regional. A nivel de discurso ambiental, esta normativa es inapelable: menciona la protección de ecosistemas autóctonos, el cuidado de paisajes naturales y culturales, la preocupación por la preservación de la Faja de Defensa Costera $(150 \mathrm{~m})$ y por su accesibilidad. Lo ambiental va unido a un desarrollo local alternativo al histórico y como componente diferencial que valoriza la nueva propuesta urbanística.

Del análisis de códigos surgen algunos elementos comunes destacados, que siguen el marco de la internalización de sseE en el mercado. El primero incluye las nuevas estrategias de от con objetivos ambientales, con el paisaje natural como elemento estructurante. Asociadas a estas, y en segundo lugar, aparecen modalidades operativas flexibles público-privadas. En tercer lugar, se establecen condiciones de edificabilidad para el aprovechamiento urbanístico privado. Otro elemento es que la conservación ambiental se asocia a la baja intensidad de uso del suelo, lo que genera escasez de ofertas y precios elevados, que finalmente apuntan a un sector de altos ingresos dispuesto a pagar esos valores (Figura 2).

FIgURA 2 Gráfico de frecuencia de citas codificadas

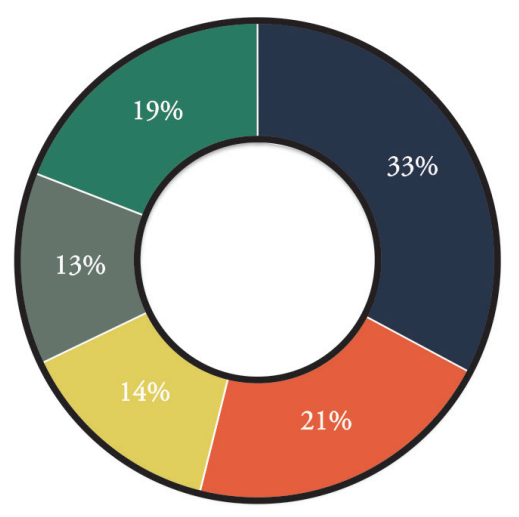

- ESTRATEgIAS DE OT CON OBJETIVO AMBIENTAL

- MODALIDADES OPERATIVAS

FLEXIBLES PÚBLICO PRIVADAS

- APROVECHAMIENTO EDILICIO

- BAJA INTENSIDAD DE USOS DEL

SUELO

- OTROS

FUENTE: ELABORACIÓN PROPIA

Como diferencias por departamentos, en Maldonado se observan los siguientes énfasis: se prevé captar las plusvalías urbanas en forma expresa (Plan Aparicio Saravia); se regula el estudio de impacto paisajístico para zonas altas como instrumento de от; se otorga el mismo valor instrumental para el plan, al componente de paisaje y patrimonio, que para aspectos infraestructurales (Plan Entre Lagunas). 
La normativa de Rocha destaca por incentivar el desarrollo urbano en los sectores con mayor grado de consolidación, a cambio de dejar cauteladas amplias zonas costeras agrestes (oc y planes derivados). Contrariamente, en Maldonado, donde hay una mayor presión inmobiliaria, la categorización de suelos que acompaña los planes reafirma usos urbanos o suburbanos sobre gran parte de los ecosistemas costeros existentes, y los nuevos planes consideran esta estructuración como inmodificable.

En ambos departamentos, la condición del aprovechamiento urbanístico es que el propietario identifique y conserve los recursos naturales y culturales del predio, ${ }^{3}$ sujeto al control de una policía ambiental o monitoreo. Vale aclarar que esta última etapa de gestión no tiene los recursos necesarios para su implementación en ninguno de los departamentos. En Rocha, la oc consagró una tasa ambiental para el desarrollo de una policía ambiental, pero hasta la actualidad nunca se implementó.

\section{La relación espacial TNE-AIA}

Se relevaron 136 casos de TNE sobre la costa de Región Este: 123 en Maldonado y 13 en Rocha. Con respecto a la superficie que el TNE ocupa en Región Este, el total de casos suma 75,6 km². En comparación al área que ocupan los padrones urbanos en Maldonado y Rocha, las áreas de TNE alcanzan un 32\%. Con respecto al área de suelo categorizado como urbano, urbano no consolidado y suburbano de la zona estudiada, la superficie de TNE representa el 10\% del total.

De acuerdo con su despliegue en territorio, de las 136 áreas TNE, 95 son barrios, 22 corresponden a condominios y 19 a edificios. En ambos departamentos los TNE tienden a concentrarse en algunas zonas, y esta concentración está relacionada también a dichas tipologías.

Los barrios y condominios en Maldonado se agrupan en sectores vinculados a espacios agrestes cercanos a los balnearios de mayor atracción (Punta Ballena, Punta del Este, Manantiales, José Ignacio) y a tramos litorales que, hasta principios del siglo xxi, estaban sin consolidar (La Boya, cercanías de José Ignacio, Garzón). La concentración de casos, sumada a la superficie que implican, genera situaciones donde las AIA y/o áreas urbanas consolidadas quedan casi totalmente encerradas por estos emprendimientos exclusivos (Figura 4).

En Rocha se encuentra la tipología de barrios, ubicada principalmente en zonas costeras muy cercanas al departamento de Maldonado, como continuación del eje Punta del Este - José Ignacio. También hay desarrollos en los alrededores de sus principales balnearios.

La tipología de edificios corresponde a fenómenos que básicamente se dan en el frente costero de barrios jardín cercanos a Punta del Este, balneario urbano por excelencia que ya cuenta con edificaciones de gran porte. Esta tipología de TNE tiene origen en la política de excepciones aplicada en Maldonado, por lo que se manifiesta como grandes hitos que distorsionan el paisaje plano y la amplitud visual fijados por la normativa (Figura 5). 
Como se aprecia en la Figura 3, los planes locales ordenan cerca de la mitad del área de estudio. Rocha tiene una mayor superficie que Maldonado bajo este régimen. A ello debe sumarse que este departamento cuenta con la oc, instrumento especialmente diseñado para una planificación integral de toda su costa. Solo 49 de los 136 casos de TNE relevados están en el área de alguno de estos cuatro planes locales, principalmente abarcadas por el plan Entre Lagunas y por el plan de Lagunas Costeras (Figura 4).

El estudio de distancias de TNE respecto a las AIA muestra claramente la ubicación estratégica de los emprendimientos: la distancia promedio entre un TNE y un AIA es de 454 metros, pudiendo variar entre 5,6 y 2.450 metros (Figura 3). El análisis por tipologías indica que el promedio de las distancias de barrios es de 520 metros; en el caso de los condominios, el resultado es de 310 metros, mientras en edificios es de 290 metros.

FIGURA 3 | Relación de proximidad entre TNE y AIA

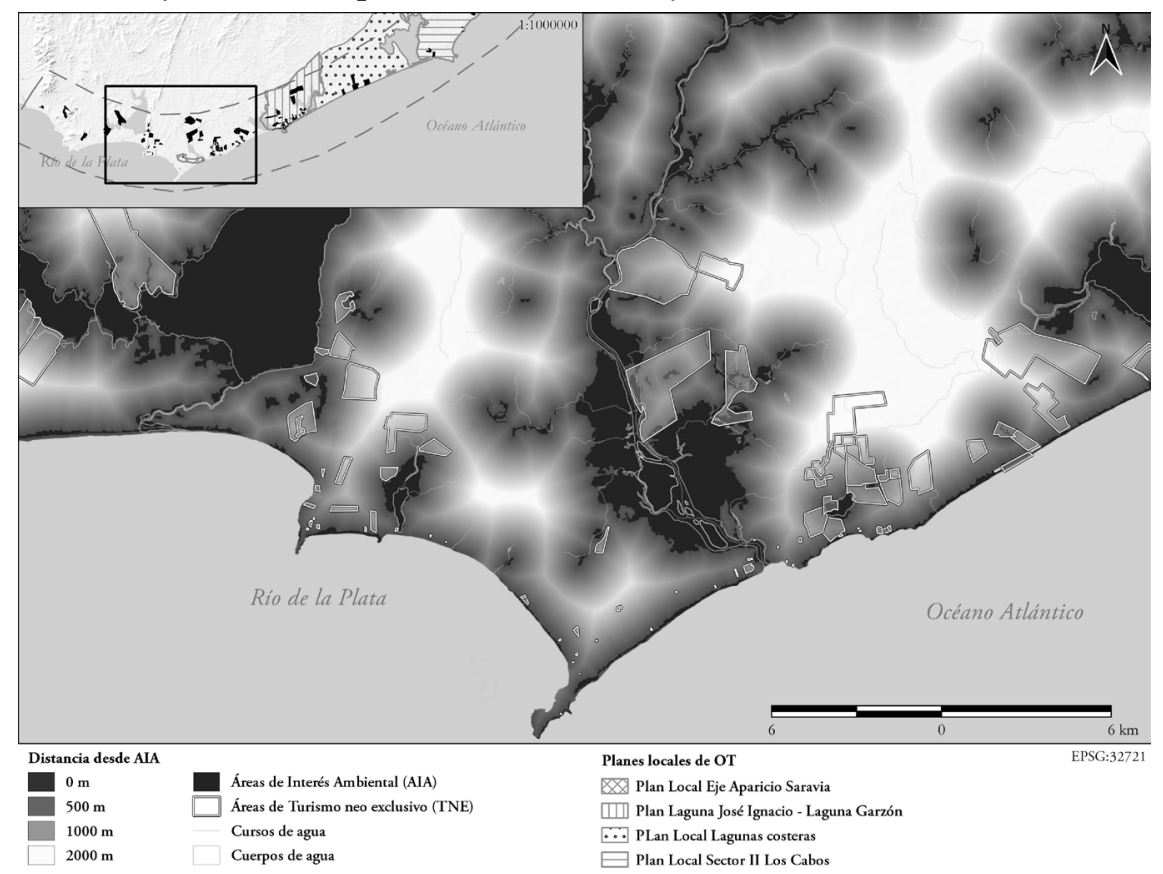

FUENTE: ELABORACIÓN PROPIA

El caso de TNE más alejado de un AIA, se encuentra entre 1.780 y 3.180 metros. Este, y solamente cuatro casos más, tienen su punto más cercano a un AIA a más de 1.000 metros.

El acercamiento es tal, que 53 de los 136 casos de TNE son limítrofes o se superponen con un AIA en una superficie aproximada a $7 \mathrm{~km}^{2}$, lo que es igual al $9 \%$ de áreas TNE. El mismo análisis, observado desde el punto de vista de los perímetros, muestra que el 13\% de los bordes del TNE se ubica frente o es interior a un AIA (Figura 4). 
FIGURA 4 | Solapamiento de área y borde de áreas de TNE sobre AIA

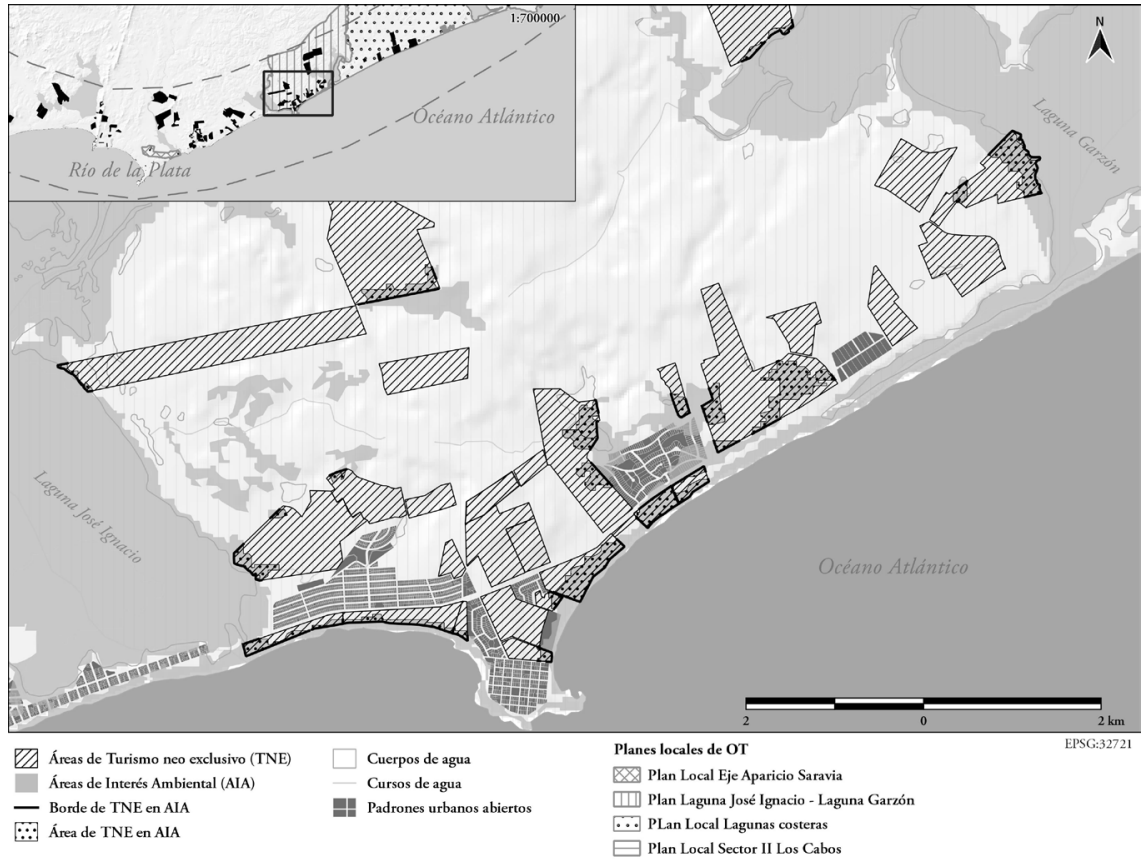

FUENTE: ELABORACIÓN PROPIA

\section{Comparación entre ofertas comerciales y solicitudes ambientales}

En el caso de los sitios de promoción comercial de los casos estudiados, las referencias a SSEE se hacen mencionando únicamente aspectos culturales (contemplación y recreación), no así otras categorías reconocidas, como soporte, abastecimiento y regulación. Desde este posicionamiento, los ecosistemas y SSEE prestados por el predio que se va a transformar y espacios cercanos, en particular espacios públicos (reserva natural, playas, vistas, brisa marina, aromas de flora autóctona y sonidos de fauna local, etcétera), se maximizan y destacan, y son ofrecidos como parte del emprendimiento asociados a las ideas de exclusividad, privilegio, calidad, inversión, distinción, estilo, seguridad y privacidad.

Por el contrario, en las solicitudes presentadas para obtener los permisos estatales para el emprendimiento (IAR, VAL y PAI), estos mismos hábitats y servicios existentes son desvalorizados, fragmentados, reducidos a su mínima expresión o directamente ignorados. De todas formas, reconociendo características naturales, el emprendimiento declara su compromiso con el cuidado ambiental del predio y se identifica como una mejora a la situación existente, la que se asocia con descuido y deterioro resultado de su condición de espacio de uso público. Una de las razones esgrimidas para la transformación de predios rurales en suburbanos o urbanos es la necesidad de incluir estos suelos en el mercado inmobiliario como forma de proteger el recurso ambiental. En el caso de la franja costera, esto se acompaña con el argumento de que el espacio en cuestión ya está sumamente alterado, por lo que varios de los 
SSEE que presta son irrelevantes y una nueva alteración del porte de la propuesta no significa mayores perjuicios.

\section{Las excepciones como política de desarrollo}

En primer lugar, debemos recalcar que, en la historia urbana de Maldonado, el uso de excepciones al от ha sido frecuente (Trochon, 2017), lo que queda manifiesto en varios edificios que superan claramente las alturas de sus entornos. La actual convocatoria oficial a solicitar excepciones (JDM, 2015), así como los expedientes ingresados y aprobados, evidencian que esta política no responde a intenciones urbanas ni territoriales, sino al fomento de la industria de la construcción, que se supone devendrá en beneficios para el sector, e indirectamente para el desarrollo local.

El periodo abierto en 2015 por un lapso de un ańo, pero mantenido por medio de sucesivas aprobaciones hasta la actualidad, otorga la exoneración del pago de tasas de edificación y del retorno por mayor valor inmobiliario, concede el aumento de metros por construir y excluye de los cálculos para tributos estos metrajes concedidos. También renueva permisos de excepciones anteriores, perimidas por no cumplimiento del plazo para su uso. En todos los casos, el ámbito legislativo departamental (JDM) recibe los documentos de los solicitantes con un agregado de la Intendencia, que consiste en un informe complementario sobre el estado de situación del sector de la construcción en el país y en Maldonado, realizado a partir de datos de la Cámara de la Construcción y el Banco de Previsión Social. Si bien las aprobaciones a estas excepciones no son por unanimidad, cuentan con votos de todos los partidos representados. En simultáneo a la apertura del periodo a excepciones, el gobierno local convoca trabajadores de otros departamentos ofreciendo empleo en estos proyectos, por lo que la cuestión se transforma rápidamente en un círculo interminable que involucra mayor convocatoria de personal, más población, más necesidad de trabajo, mayor necesidad de excepciones (Sciandro \& Zeballos, 2019).

A raíz de la especificidad en la activación del empleo y las inversiones, y no en aspectos territoriales, esta política genera la apertura a todo tipo de solicitudes y con posibilidad de las más diversas ubicaciones. Los permisos otorgados avalan desde septuplicar la cantidad de pisos posibles, ocupar espacios destinados a retiros, hasta el traspaso de calles públicas al ámbito privado. A mayo de 2019 existían 58 expedientes aprobados, mayoritariamente ubicados en primera línea frente al mar o en el espacio playa y, según las características de los proyectos, 19 son obras destinadas al TNE, tanto en la modalidad de edificio como de condominio. De acuerdo con el estudio de la DinOt (2018) sobre desvíos autorizados en altura o en el factor de ocupación total (Fот), uno de estos 19 casos tiene 327\% de desvío otorgado (Fendi Chateau), dos superan el 400\% (The Colette y Paramount) y uno alcanza el $647 \%$ (Venetian Tower) (Figura 5). 
FIGURA 5 | Arriba: tipología condominio The Colette. Abajo: tipología edificio en primera línea frente al mar Venetian Tower, Fendi Chateau y Paramount
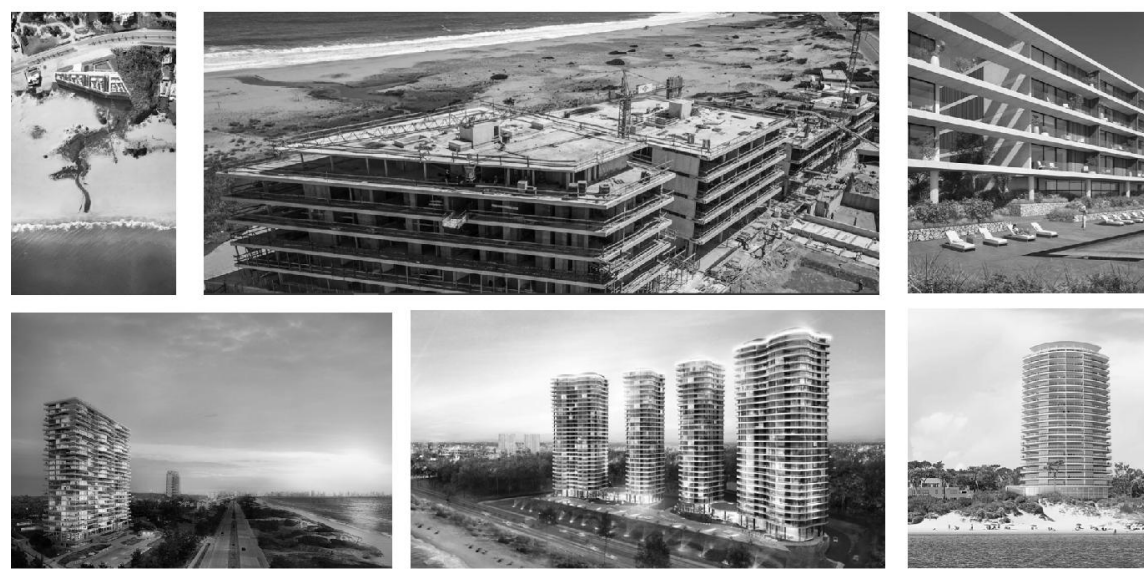

FUENTE: IMÁGENES EXTRAÍDAS DE PÁGINAS DE PROMOCIÓN

\section{Discusión}

Los gobiernos departamentales de Maldonado y Rocha han aprobado instrumentos de diversa índole para adecuarse al nuevo marco normativo nacional, que a la vez retoma tendencias globales en pro de un desarrollo sustentable. A nivel instrumental, intentan traspasar fragmentaciones previas con nuevos instrumentos, pero siguiendo una línea neoliberal que emplea conceptos fundantes basados en la necesidad de regularizar la naturaleza, para introducirla en el mercado y así conservarla.

Al igual que en otros ejemplos de América Latina, aquí se conjugan cuatro variables: el cuidado ambiental, la construcción como motor de desarrollo, la baja intensidad de usos del suelo y la apuesta a propietarios de altos ingresos. Estas variables se retroalimentan en el discurso: la conservación de componentes naturales se convierte en el fundamento del desarrollo inmobiliario selecto del que depende su cuidado, mientras los ambientes agrestes se ven como espacios que deben incorporarse al mercado, pues son oportunidades no usadas hasta el momento para el desarrollo económico regional. La planificación relaciona la necesidad de protección ambiental con la urgencia de empleo local, y considera al mercado como solución a ambas cuestiones.

Por su lado, la industria turística asociada a la inmobiliaria intenta seguir compitiendo, ofertando cada vez mayores condiciones de exclusividad y naturaleza, aunque su estrategia es la transformación sistemática de espacios naturales. Para seguir siendo atractivo como destino de viaje o inversión, en la medida en que la costa se vuelve un sitio de turismo masivo y urbanizado, el TR avanza sobre el territorio de tres formas distintas: extendiéndose en el litoral sin consolidar; sobre 
espacios cercanos al litoral, vinculados preferentemente a hitos naturales; y sobre el frente costero de centros urbanos.

En cualquiera de estas modalidades el TNE tiene un rol protagónico, a pesar de los numerosos llamados de atención acerca de la brecha que genera en la sociedad, la artificialización del territorio y la pérdida de control del Estado sobre su espacio y recursos. En Región Este, el TNE equivale actualmente a un tercio del área urbana abierta y acapara sitios que resultan fundamentales para el mantenimiento de SSEE clave para toda la población, como conservación de ecosistemas en peligro, prevención de erosión costera, abastecimiento de agua potable o resguardo de información arqueológica. Igualmente restringe el acceso público a paisajes apreciados por toda la comunidad, y usa y presiona centros urbanos consolidados y núcleos históricos. La planificación vigente habilita aun mayores transformaciones en el mismo sentido. Asume dichos riesgos a cambio de modalidades residenciales que apuestan a la caricaturización de zonas de interés ambiental para el ocio estival de los sectores más pudientes, con un ciclo de vida que depende de tendencias globales.

A lo anterior se suman procesos que favorecen la mala aplicación de la planificación. Uno de ellos es que, en las etapas de revisión y autorización de propuestas, la información ambiental es usada a discreción y/o distorsionada por emprendedores y consultoras, y los instrumentos de aprobación consienten este uso.

Asimismo, la estructuración de la norma y el territorio, que aplica cuestiones concretas y objetivables a ciclos naturales dinámicos y/o impredecibles, genera la dilución de los objetivos de sustentabilidad. Ejemplo es el límite histórico de 150 metros de exclusión sobre la línea de ribera, que por un lado es garantía de cuidado y, por otro, un permiso para urbanizar todo lo que está más allá de su trazado, aunque claramente sea parte de la misma unidad geomorfológica y se necesite para mantener el recurso playa. Maldonado mantiene este límite de manera indiscutida, mientras que Rocha amplió esta exclusión a extensas zonas de la costa, pero cabe señalar que no ha recibido presión fuerte del mercado para estos espacios.

A la fragmentación fundacional que significa la división del territorio en miles de predios privados, se suma la fragmentación acumulativa conceptual del sistema que se quiere proteger en el proceso de aprobación de emprendimientos. En la primera etapa y más general, a nivel de planes, se establecen cuestiones sistémicas e integrales. Luego, para habilitar proyectos de fraccionamiento se contempla principalmente la categorización de suelo, si bien los proyectos presentan declaraciones de cuidado del entorno ecosistémico. Vale aclarar que la mayoría de estas declaraciones se contradice en la propuesta, planteando desarrollar inmobiliariamente zonas de fragilidad ecosistémica y/o quebrando la planificación territorial existente. Finalmente, la aprobación de cada vivienda depende de un Informe Ambiental, documentación con énfasis en aspectos ingenieriles y datos de componentes naturales a escala de padrón (Gadino \& Taveira, 2020), lo que lo despoja de toda posibilidad de lectura ecosistémica. El análisis de impactos acumulativos es mencionado en la LOTDS (2008) y recientemente recuperado en la Directriz Costera (2019), pero su aplicación no se observa a nivel territorial.

Finalmente, para cuando la planificación y formas de gestión establecidas no conformen el mercado y/o sean entendidas como trabas al desarrollo, se prevén 
mecanismos de excepción que dan lugar a la demanda. En Maldonado, que es donde se da la mayor presión inmobiliaria y donde existe más población estable que depende principalmente de la actividad de la construcción, abandonar los acuerdos formulados sobre usos del territorio para satisfacer la promoción privada es una política reiterada.

\section{Conclusiones}

La estrategia empleada por el actual от intenta aportar, en una única fórmula, a dos cuestiones fundamentales: la protección del ambiente y la economía nacional. En el caso de la primera, se aplica la premisa neoliberal de que, para proteger espacios naturales, estos deben ingresar al mercado y tener usos poco intensivos. En el actual modelo esto resulta en la fórmula del TNE de ofrecer áreas de interés ambiental como un bien escaso, para ser disfrutadas y cuidadas a los que las pueden pagar, recortando el acceso al resto de la población, incluido el contralor del Estado.

Para el segundo problema, se recurre nuevamente a la promoción de la industria de la construcción, pues, de tener éxito, logra un impacto positivo (al menos a corto plazo) en toda la columna socioeconómica local. Sin embargo -como muestran los resultados de este trabajo y de otros citados sobre la región-, sus repercusiones negativas en paisajes, ecosistemas y servicios asociados llegan a ser graves y en gran medida irreversibles. Como sucede históricamente, las inversiones se asocian principalmente a intereses privadoempresariales internacionales, que en el actual contexto son sumamente volátiles y exigen cada vez mayores otorgamientos para su concreción.

El grado máximo de esta fórmula es la política de excepciones, cuyas consecuencias impactan en los sSEe de los sectores más destacados del litoral nacional. Por otro lado, degrada el largo trabajo de planificación consensuada, colaborando a instalar la lógica de la oportunidad individual por sobre las políticas públicas. Impone el éxito económico a corto plazo, cediendo derechos de la comunidad y desatendiendo todos los factores establecidos para el cuidado del entorno natural y cultural. Si bien este caso no es el único de otorgamientos en el país, se destaca por su permanencia y amplia aprobación, al haber sido desarrollado en un marco creciente de normativa territorial.

Si bien este caso no es el único de otorgamientos en el país, se destaca por su permanencia y amplia aprobación, desarrollado en un marco creciente de normativa territorial.

Frente a estas constataciones, la cuestión parece tener varios caminos para recorrer. Algunos son referidos a aspectos instrumentales y seguramente ajustables a corto o mediano plazo. La legislación es cada vez mayor y más integrada, y cuenta con una vasta cantidad de nuevos instrumentos aún sin usar, que auspician un mejor escenario de gestión (p.e., implementar tasas ambientales y cobro de plusvalías, considerar impactos acumulativos, ampliar límites de franja costera, atender al paisaje y al patrimonio natural y cultural, realizar inventarios y monitoreo ambiental, sistematizar la información, profundizar procesos de participación).

El mayor desafío parece ser trabajar mancomunadamente sobre cuestiones de base, reflexionando sobre si las urgencias actuales valen el riesgo futuro, qué oportunidades existen aún en este contexto de microfragmentación privada del espacio, 
o si la dupla turismo-construcción es la única fórmula aplicable en este territorio, a pesar de causar grandes pérdidas en los recursos y bienes comunes que sostienen casi todas las actividades que se dan sobre él.

\section{Referencias bibliográficas}

Aizcorbe, M., Fernández Bouzo, S. \& Wertheimer, M. (2014). Moros en la costa. Ambiente, actores locales y conflicto en torno a los megaproyectos de urbanización sobre la franja costera de los partidos de Avellaneda-Quilmes y Vicente López (2000-2011). En G. Merlinsky (Comp.), Cartografías del conflicto ambiental en Argentina. Fundación ciccus (Centro de Integración, Comunicación, Cultura y Sociedad).

Aledo, A. (2008). De la tierra al suelo: la transformación del paisaje y el nuevo turismo residencial. Arbor, 184(729), 99-113. https://doi.org/10.3989/arbor.2008.i729.164

Aledo, A. (2016). Turismo residencial y vulnerabilidad en el interior del Levante español. En J. Gascón \& E. Cañada (Coords.), Turismo residencial y gentrificación rural (pp. 37-59). pasos, Revista de Turismo y Patrimonio Cultural, Colección pasos edita, $n^{\circ} 5$ / Foro de Turismo Responsable, Colección Thesis 5. http://www.pasosonline.org/Publicados/ pasosoedita/PSEdita_16_Definitivo_e-book.pdf

Alonso, E. \& Bassagoda, M. J. (2002). La vegetación costera del SE uruguayo: ambientes y biodiversidad. Documentos de divulgación Museo Nacional de Historia Natural y Antropología, Uruguay (julio 2002, $\mathrm{n}^{\circ}$ 5). https://www.mnhn.gub.uy/innovaportal/ file/3419/1/La_vegetacion.pdf

Álvarez, A., Blum, A. \& Gallego, F. (2015). Atlas de Cobertura del Suelo del Uruguay. Cobertura del suelo y detección de cambios 2000-2011. Land Cover Classification System. http:// www.fao.org/3/a-i4372s.pdf

Babinger, F. (2012). El turismo ante el reto de peligros naturales recurrentes: una visión desde Cancún. Investigaciones geográficas, (78), 75-88. https://doi.org/10.14350/rig.32471

Baptista, E., Domareski Ruiz, T. \& dos Anjo, F. (2018). A ocupaçáo urbana no Litoral Norte do Rio Grande do Sul, Brasil, e suas implicaçóes no turismo de segunda residências. URBE. Revista Brasileira de Gestão Urbana,10(2). https://doi.org/10.1590/21753369.010.002.AO03

Barboza Núńez, E. (2019). Litorales imaginados, dominios construidos: desarrollo turístico de sol y playa y discurso colonial en Guanacaste, Costa Rica. Tesis doctoral en Estudios de la Sociedad y la Cultura, Universidad de Costa Rica.

Beck, U. (2013). La sociedad del riesgo: En camino hacia otra sociedad moderna. Grupo Planeta.

Boretto, G., Rouzaut, S., Cioccale, M., Gordillo, S. \& Benítez, Y. (2018). Dinámica costera y antropización en playas uruguayas. Un análisis integrado para su conservación. Revista Mexicana de Ciencias Geológicas, 35(3), 291-306. http://dx.doi.org/10.22201/ cgeo.20072902e.2018.3.865

Brockington, D. \& Igoe, J. (2006). Eviction for conservation. A global overview. Conservation and Society, 4(3), 424-470. https://www.jstor.org/stable/26396619 
Brum Bulanti, L., de Álava, D., Chocca, Y. \& Marín, Y. (2020). De espaldas al mar. Desafíos para un manejo integrado del patrimonio arqueológico costero y marítimo del Uruguay. Revista Costas, 2(1), 81-104. http://doi.org/10.26359/costas.0502

Camacho Lomelí, R. (2015). Urbanización turístico-costera desigual en Playa del Carmen, Quintana Roo (México). GeoGraphos, 6(77), 107-134. http://dx.doi.org/10.14198/ GEOGRA2015.6.77

Castree, N. (2006). From neoliberalism to neoliberalisation: Consolations, confusions, and necessary illusions. Environment and Planning A, 38, 1-6. https://doi. org $/ 10.1068 \% 2 \mathrm{Fa} 38147$

Castro Casas, C. (2018). La Ley de Ordenamiento Territorial y Desarrollo Sostenible. A 10 años del inicio del proceso de institucionalización del ordenamiento territorial. Cuadernos del CLAEH, 37(108), 189-206. https://doi.org/10.29192/CLAEH.37.2.9

Clement, R. \& Grant, J. (2012). Enclosing Paradise: The design of gated communities in Barbados. Journal of Urban Design, 17(1), 43-60. https://doi.org/10.1080/1357480 9.2011 .646249

Conde, D. (2014). Costas. Nuestro tiempo, 09. Comisión del Bicentenario. Montevideo, Uruguay, 2013-2014.

Corbin, J. \& Strauss, A. (1990). Grounded theory research: Procedures, canons and evaluative criteria. Qualitative Sociology, 13, 3-21. https://sites.duke.edu/niou/files/2014/07/ W10-Corbin-and-Strauss-grounded-theory.pdf

Daniel, T. C., Muhar, A., Arnberger, A., Aznar, O., Boyd, J. W., Chan, K. A., Costanza, R., Elmqvist, T., Flint, C., Gosber, P., Gret-Regamey, A., Lave, R., Muhar, M., Ribe, R., Schauppenlehner, T., Sikor, T., Solovy, I., Spjerenbirg, M., Taczanowska, K. \& Von Der Dunk, A. (2012). Contributions of cultural services to the ecosystem services agenda. Proceedings of the National Academy of Sciences of the United States of America. National Academy of Sciences. https://doi.org/10.1073/pnas.1114773109

de Álava, D. (2008). Proyecto de restauración sistema de playas y dunas frontales de la bahía de Portezuelo. Unión de Vecinos de Punta Ballena y Laguna del Diario. http://dx.doi. org/10.13140/RG.2.2.16197.68323

De Matheus e Silva, L. F., Zunino, H. M. \& Huiliñir Curío, V. (2018). El negocio de la conservación ambiental: cómo la naturaleza se ha convertido en una nueva estrategia de acumulación capitalista en la zona andino lacustre de Los Ríos, sur de Chile. Scripta Nova. Revista Electrónica de Geografía y Ciencias Sociales, 22. https://doi.org/10.1344/ sn2018.22.19021

Demajorovic, J., Aledo, A., Landi, B. \& Mantovani, A. (2011). Complejos turísticos residenciales. Análisis del crecimiento del turismo residencial en el Mediterráneo español y en el Litoral Nordestino (Brasil) y su impacto socio-ambiental. Estudios y Perspectivas en Turismo, 20(4), 772-796. https://www.redalyc.org/articulo.oa?id=180722696002

Díaz Pellicer, L. (2012). El turismo receptivo en Uruguay (1930-1986). Documento online no 27/Reedición Febrero 2012 Documentos de trabajo (Programa de Historia Económica y Social, Unidad Multidisciplinaria, Facultad de Ciencias Sociales, Universidad de la República, Uruguay). https://www.colibri.udelar.edu.uy/jspui/ handle/20.500.12008/4671 
Dirección Nacional de Ordenamiento Territorial (DiNOT). (2011). Estrategias Regionales de Ordenamiento Territorial. Ministerio de Vivienda, Ordenamiento Territorial y Medio Ambiente (мvотма), Uruguay.

Dirección Nacional de Ordenamiento Territorial (DINOT). (2018). Análisis de la política de exoneración tributaria y flexibilización urbana en el departamento de Maldonado. Ministerio de Vivienda, Ordenamiento Territorial y Medio Ambiente (мvoтмA), Uruguay.

Directriz Costera. (2019). Ley Nacional 19.772. https://www.impo.com.uy/bases/ leyes/19772-2019

Domínguez, J. A. \& Aledo, A. (2005). Turismo residencial y sostenibilidad: el caso de la costa sur-occidental española. En T. Mazón \& A. Aledo (Eds.), Turismo residencial y cambio social (pp. 517-534). Universidad de Alicante, Aguaclara, España. https://dialnet. unirioja.es/servlet/articulo?codigo $=1408026$

Durand Smith, L., Figueroa Díaz, F. \& Guzmán Chávez, M. G. (2011). La ecología política en México ¿Dónde estamos y para dónde vamos? Estudios Sociales, 19(37), 281-307. www.scielo.org.mx/scielo.php?script=sci_arttext\&pid=S0188-45572011000100011

El Observador. (2018, abril 16). Construcción en Maldonado con menor nivel de mano de obra en 12 ańos. El Observador [Uruguay]. https://www.elobservador.com. uy/nota/construccion-en-maldonado-con-menor-nivel-de-mano-de-obra-en-12anos-2018416500

Escobar, A. (1995). Encountering development. The making and unmaking of the Third World. Princeton University Press.

Escobar, A. (2000). El lugar de la naturaleza y la naturaleza del lugar: globalización y posdesarrollo. En A. Viola (Ed.), Antropología del desarrollo. Teorías y estudios etnográficos en América Latina (pp. 169-216). Paidós Studio.

Fairhead, J., Leach, M. \& Scoones, I. (2012). Green Grabbing: A new appropriation of nature? Journal of Peasant Studies, 39(2), 237-261. https://doi.org/10.1080/03066150.2012. 671770

Félix, A. G. \& García, N. (2020). Estudio de pérdidas y estrategias de reactivación para el sector turístico por crisis sanitaria Covid-19 en el destino Manta (Ecuador). Revista Internacional de Turismo, Empresa y Territorio, 4(1), 79-103. https://doi.org/10.21071/ riturem.v4i1.12743

freplata. (2005). Programa de Acción Estratégico. Protección ambiental del Río de la Plata y su frente maritimo. Prevención y control de la contaminación y restauración de hábitats. Proyecto PNUD/GEF - RLA/99/G3I.

Gadino, I., Brazeiro, A., Panario, D., Roche, I. \& Gutiérrez, O. (2012). El modelo actual de desarrollo turístico al oeste del balneario La Paloma, Rocha, Uruguay. Tendencias, riesgos y propuestas. Sustentabilidade em Debate, 3(2), 21-40. http://dx.doi. org/10.18472/SustDeb.v3n2.2012.8125

Gadino, I. \& Taveira, G. (2020). Ordenamiento y gestión del territorio en zonas costeras con turismo residencial. El caso de Región Este, Uruguay. Revista de Geografía Norte Grande, (77), 233-251. http://ojs.uc.cl/index.php/RGNG/article/view/29201 
García de Fuentes, A., Jouault, S. \& Romero, D. A. (2019). Representaciones cartográficas de la turistificación de la península de Yucatán a medio siglo de la creación de Cancún. Investigaciones Geográficas [Instituto de Geografía, UnAM], (100). https://doi. org/10.14350/rig.60023

García, R. (2019). La expansión de los barrios privados en el Uruguay. Colab.: M. Rodríguez, M. Rovira \& M. Terra. Dirección Nacional de Ordenamiento Territorial (DINOT), Ministerio de Vivienda y Ordenamiento Territorial (мVOTMA). https://sit.mvotma. gub.uy/pdf/BBPP_Uruguay.pdf

Gómez-Baggethun, E., De Groot, R., Lomas, P. \& Montes, C. (2010). The history of ecosystem services in economic theory and practice: from early notions to markets and payment schemes. Ecological Economics, 69(6), 1209-1218. https://doi.org/10.1016/j. ecolecon.2009.11.007

Harvey, D. (1989). From managerialism to entrepreneurialism. The transformation in urban governance in late capitalism. Geografiska Annaler. Series B, Human Geography, 71(1), 3-17. https://doi.org/10.2307/490503

Harvey, D. (2014). Diecisiete contradicciones y el fin del capitalismo. Instituto de Altos Estudios Nacionales del Ecuador (IAEN) y Traficantes de sueños. https://www.traficantes.net/ sites/default/files/pdfs/Diecisiete\%20contradicciones\%20-\%20Traficantes\%20 de\%20Sue\%C3\%B1os.pdf

Hernández, F. M. (2010). La neoexclusividad turística en el litoral marítimo bonaerense: Nuevas prácticas, nuevos escenarios, nuevos paisajes. VI Jornadas de Sociología de la UNLP, 9 y 10 de diciembre de 2010, La Plata, Argentina. En Memoria Académica. http://www.memoria.fahce.unlp.edu.ar/trab_eventos/ev.5777/ev.5777.pdf

Hidalgo, R., Volker, P. \& Ramírez, N. (2014). La ciudad inmobiliaria: Mecanismos institucionales y relaciones de poder. El caso del Área Metropolitana de Valparaíso. Scripta Nova. Revista Electrónica de Geografía y Ciencias Sociales, 18 (Número especial dedicado al xiII Coloquio Internacional de Geocrítica: El control del espacio y los espacios de control), 1-19. https://revistes.ub.edu/index.php/ScriptaNova/article/ view/15023

Igoe, J. \& Brockington, D. (2007). Neoliberal conservation: A brief introduction. Conservation and Society, 5(4), 432-449.

Instituto Nacional de Estadística y Censo (INE), Uruguay. (2011). Censo 2011. https://www. ine.gub.uy/censos-2011

Jordan, A., Wurzel, R. K. W. \& Zito, A. R. (Eds.). (2003). New instruments of Environmental Governance? National experiences and prospects. Routledge.

Junta Departamental de Maldonado (JDM), Uruguay. (2015). Decreto 3.941 de 2015-10-14. https://www.juntamaldonado.gub.uy/index.php/decretos-2013/1841-3941.html

Knuth, S. (2016). Seeing green in San Francisco: City as resource frontier. Antipode, 48(3). https://doi.org/10.1111/anti.12205

Kranjčević, J. \& Hajdinjak, S. (2019). Tourism urbanization in Croatia. The cases of Poreč in Istria and Makarska in Dalmatia. Südosteuropa, 67(3). https://doi.org/10.1515/soeu2019-0028

Lang, R. E. \& Danielsen, K. A. (1997). Gated communities in America: Walling out the world? Housing Policy Debate, 8(4), 867-877. https://doi.org/10.1080/10511482.1997.9521 
Leff, E. (2015). The power-full distribution of knowledge in political ecology: a view from the South. En T. Perreault, G. Bridge \& J. McCarthy (Eds.), Routledge Handbook of Political Ecology (pp. 64-75). Routledge.

Ley de Centros Poblados $n^{\circ} 10.723$ de 21/04/1946, modificado por Ley $\mathrm{n}^{\circ} 10.866$ del 15/10/1946, que complementa y sustituye artículos de la referida. https://www.impo. com.uy/bases/leyes/10723-1946

Ley de Ordenamiento Territorial y Desarrollo Sostenible (LOTDS), no 18.308 del 18/06/2008. https://www.impo.com.uy/bases/leyes/18308-2008

March, H. (2013). Neoliberalismo y medio ambiente: una aproximación desde la geografía crítica. Documents d'Anàlisi Geogràfica, 59(1), 137-153. http://dx.doi.org/10.5565/ rev/dag. 17

McAfee, K. (1999). Selling nature to save it? Biodiversity and Green developmentalism. Environment and Planning D: Society and Space, 17(2), 133-154. https://doi. org/10.1068\%2Fd 170133

McCarthy, J. \& Prudham, S. (2004). Neoliberal nature and the nature of neoliberalism. Geoforum, 35(3), 275-283. http://dx.doi.org/10.1016/j.geoforum.2003.07.003

Mendonça Ferreira, A. \& Vilas Boas, E. (2020). A ética de Emmanuel Lévinas e a ausência de alteridade na instituição das gated communities: quem ou o que está por detrás dos muros? Revista de Direito Urbanistico, Cidade e Alteridade, 6(1), 118-134. http:// dx.doi.org/10.26668/IndexLawJournals/2525-989X/2020.v6i1.6543

Millennium Ecosystem Assessment (MEA). (2005). Ecosystems and human well-being. Synthesis. A report of the Millennium Ecosystem Assessment. Island Press. https://www. millenniumassessment.org/documents/document.356.aspx.pdf

Ministerio de Turismo (mintur), Uruguay. (2018). Anuario 2018 - Estadísticas de Turismo. https://www.gub.uy/ministerio-turismo/comunicacion/publicaciones/anuario-2018

Ministerio de Turismo (MINTUR), Uruguay (2021). MINTUR presentó los datos oficiales del turismo en Enero 2021 y la creación del Observatorio Turístico Nacional. https://www.gub. $\mathrm{uy} / \mathrm{ministerio-turismo/comunicacion/noticias/mintur-presento-datos-oficiales-del-}$ turismo-enero-2021-creacion-del

Morell, S. \& Membrado-Tena, J. (2019). Causas y consecuencias del crecimiento urbanístico en el litoral valenciano a través de la evolución de los usos del suelo. El caso de Oliva. Cuadernos de Turismo, (44), 303-326. http://dx.doi.org/10.6018/turismo.44.404861

Nagy, G., Bidegain, M., Verocai, J. \& de los Santos, B. (2016). Escenarios climáticos futuros sobre Uruguay. Basados en los nuevos escenarios socioeconómicos RCP. Ministerio de Vivienda y Ordenamiento Territorial (мvотмA), División de Cambio Climático, Uruguay.

Oficina de Planeamiento y Presupuesto (OpP), Organización de los Estados Americanos (oEA) \& Banco Interamericano de Desarrollo (BID). (1992). Uruguay. Estudio ambiental nacional. https://www.oas.org/dsd/publications/Unit/oea10s/begin.htm

Panario, D. \& Gutiérrez, O. (2005). La vegetación en la evolución de playas arenosas. El caso de la costa uruguaya. Ecosistemas, Revista cientifica y técnica de ecología y medio ambiente, 14(2), 150-161. http://dx.doi.org/10.7818/re.2014.14-2.00

Peck, J. \& Tickell, A. (2002). Neoliberalizing space. Antipode, 34(3), 380-404. https://doi. org/10.1111/1467-8330.00247

Peet, R. \& Watts, M. (Eds.). (2002). Liberation ecologies ( $2^{\text {nd }}$ ed.). Routledge. 
Peluso, N. L. \& Lund, C. (2011). New frontiers of land control: Introduction. Journal of Peasant Studies, 38(4), 667-681. https://doi.org/10.1080/03066150.2011.607692

Pitarch-Garrido, M. D. (2020). Turismo y vulnerabilidad territorial: capacidad de resiliencia de los diferentes modelos turísticos frente a la crisis pandémica del coronavirus en Espańa. En M. R. Simancas Cruz, R. Hernández Martín \& N. Padrón Fumero (Coords.), Turismo pos-COVID-19. Reflexiones, retos y oportunidades (pp. 211-223). Universidad de La Laguna, Espańa. https://doi.org/10.25145/b.Turismopos-COVID-19.2020

Pontes, M. A., García-Marín, R. \& Moreno-Muñoz, D. (2020). Turismo, producción inmobiliaria y procesos espaciales: la difusión del modelo turístico español hacia Brasil. Revista EURE - Revista de Estudios Urbano Regionales, 46(137), 135-156. http://dx.doi. org/10.4067/S0250-71612020000100135

Robayna, A. (2009). Programa ECOPLATA. Presión antrópica en la costa uruguaya. Análisis de indicadores sobre turismo y transporte. Informe de resultados del proyecto PNUD URU 06/016, "Conectando el conocimiento con la acción para la gestión integrada de la zona costera uruguaya del Río de la Plata”. https://bit.ly/2VgXIIz

Robbins, P. (2012). Political ecology: a critical introduction to Geography (2 ${ }^{\text {nd }}$ ed.). WileyBlackwell.

Roche, I. \& Gadino, I. (2018). Los atractivos turísticos y el desarrollo territorial en la costa uruguaya. Topofilia, Revista de Arquitectura, Urbanismo y Territorios (Instituto de Ciencias Sociales y Humanidades "Alfonso Vélez Pliego"), 11(17), 32-55. http://69.164.202.149/topofilia/index.php/topofilia/article/view/28

Roitman, S. (2013). Close but divided: How walls, fences and barriers exacerbate social differences and foster urban social group segregation. Housing, Theory and Society, 30(2), 156-176. https://doi.org/10.1080/ 14036096.2012.728150

Sciandro, J. \& Zeballos, L. (2019). Excepciones como instrumento de planificación en Maldonado. Tekoporá, Revista Latinoamericana de Humanidades Ambientales y Estudios Territoriales, 1(1), 171-180. https://revistatekopora.cure.edu.uy/index.php/reet/ article/view/23

Scott, J. (1998). Seeing like a State: How certain schemes to improve the human condition have failed. Yale University Press.

Sousa, P. G. de, Esdras Matheus, M. \& Fragoso, V. (2016). Do turismo residencial aos complexos turísticos imobiliários: a apropriação da zona costeira do nordeste brasileiro pela atividade turística imobiliária. Ambiente \& Sociedade, 19(3), 177-198. https://doi. org/10.1590/1809-4422ASOC141673V1932016

Soutullo, A., Clavijo, C. \& Martínez-Lanfranco, J. A. (Eds.). (2013). Especies prioritarias para la conservación en Uruguay. Vertebrados, moluscos continentales y plantas vasculares. SNAP / DINAMA / MOTMA y DICYT / MEC. https://www.researchgate.net/ publication/280601164_Especies_prioritarias_para_la_conservacion_en_Uruguay

Svampa, M. (2005). Los que ganaron. La vida en los countries y barrios privados. Biblos.

Trochon, Y. (2017). Punta del Este. El edén oriental. 1907-1997. Editorial Fin de Siglo.

Van Noorloos, F. (2015). Tourism turning real estate: How to deal with residential tourism investment in the Global South? LANDac Policy Brief, 01, 1-8. https://doi. org/10.13140/RG.2.2.27125.47846 
Varela, A. (2017). Paraisos exclusivos. Emprendimientos turistico-residenciales cerrados emergentes en Maldonado. Tesis de Maestría en Ordenamiento Territorial y Desarrollo Urbano, Facultad de Arquitectura, Diseño y Urbanismo, Universidad de la República, Uruguay. https://www.colibri.udelar.edu.uy/jspui/handle/20.500.12008/20952

Zielinski, S. \& Botero, C. (2020). Beach tourism in times of covid-19 pandemic: Critical issues, knowledge gaps and research opportunities. International Journal of Environmental Research and Public Health, 17(19), 7288. https://doi.org/10.3390/ijerph17197288 